\title{
STAT3 exacerbates survival of cancer stem-like tumorspheres in EGFR-positive colorectal cancers: RNAseq analysis and therapeutic screening
}

Chun-Chia Cheng ${ }^{1,2+}$, Po-Nien Liao ${ }^{1,2+}$, Ai-Sheng Ho ${ }^{3+}$, Ken-Hong Lim ${ }^{1,2,7}$, Jungshan Chang ${ }^{4}$, Ying-Wen Su ${ }^{1,2,7}$, Caleb Gon-Shen Chen ${ }^{1,2,7}$, Ya-Wen Chiang 1,2, Bi-Ling Yang ${ }^{3}$, Huan-Chau Lin ${ }^{1,2}$, Yu-Cheng Chang ${ }^{1,2}$,

Chun-Chao Chang ${ }^{5,6^{*}}$ and Yi-Fang Chang ${ }^{1,2,7^{*}}$

\section{Abstract}

Background: Cancer stem cells are capable of undergoing cell division after surviving cancer therapies, leading to tumor progression and recurrence. Inhibitory agents against cancer stem cells may be therapeutically used for efficiently eradicating tumors. Therefore, the aim of this study was to identify the relevant driver genes that maintain cancer stemness in epidermal growth factor receptor (EGFR)-positive colorectal cancer (CRC) cells and to discover effective therapeutic agents against these genes.

Methods: In this study, EGFR-positive cancer stem-like cells (CSLCS) derived from HCT116 and HT29 cells were used as study models for in vitro inductions. To identify the differential genes that maintain CSLCs, RNAseq analysis was conducted followed by bioinformatics analysis. Moreover, a panel containing 172 therapeutic agents targeting the various pathways of stem cells was used to identify effective therapeutics against CSLCS.

Results: RNAseq analysis revealed that 654 and 840 genes were significantly upregulated and downregulated, respectively, in the HCT116 CSLCs. Among these genes, notably, platelet-derived growth factor A (PDGFA) and signal transducer and activator of transcription 3 (STAT3) were relevant according to the cancer pathway analyzed using NetworkAnalyst. Furthermore, therapeutic screening revealed that the agents targeting STAT3 and Wnt signaling pathways were efficient in reducing the cell viabilities of both HCT116 and HT29 cells. Consequently, we discovered that STAT3 inhibition using homoharringtonine and STAT3 knockdown significantly reduced the formation and survival of HT29-derived tumorspheres. We also observed that STAT3 phosphorylation was regulated by epidermal growth factor (EGF) to induce PDGFA and Wnt signaling cascades.

Conclusions: We identified the potential genes involved in tumorsphere formation and survival in selective EGFRpositive CRCs. The results reveal that the EGF-STAT3 signaling pathway promotes and maintains CRC stemness. In addition, a crosstalk between STAT3 and Wnt activates the Wnt/ $\beta$-catenin signaling pathway, which is also responsible for cancer stemness. Thus, STAT3 is a putative therapeutic target for CRC treatment.

Keywords: Cancer stem cell, EGFR, LGR5, PDGFA, STAT3, Wnt

\footnotetext{
* Correspondence: chunchao@tmu.edu.tw; changyifang@gmail.com

${ }^{\dagger}$ Chun-Chia Cheng, Po-Nien Liao and Ai-Sheng Ho contributed equally to this work.

${ }^{5}$ Division of Gastroenterology and Hepatology, Department of Internal

Medicine, Taipei Medical University Hospital, Taipei, Taiwan

'Division of Hematology and Oncology, Department of Internal Medicine,

Mackay Memorial Hospital, Taipei, Taiwan

Full list of author information is available at the end of the article
}

(c) The Author(s). 2018 Open Access This article is distributed under the terms of the Creative Commons Attribution 4.0 International License (http://creativecommons.org/licenses/by/4.0/), which permits unrestricted use, distribution, and reproduction in any medium, provided you give appropriate credit to the original author(s) and the source, provide a link to the Creative Commons license, and indicate if changes were made. The Creative Commons Public Domain Dedication waiver (http://creativecommons.org/publicdomain/zero/1.0/) applies to the data made available in this article, unless otherwise stated. 


\section{Background}

Colorectal cancer (CRC) is a leading cause of cancer-related morbidity and mortality worldwide. Early diagnosis followed by surgery can cure patients with CRC. Adjuvant chemotherapy is used for eradicating remnant tumor cells in high-risk CRC (stages II and III). Adjuvant therapy consists of either a single agent, such as capecitabine or 5FU/leucovorin, or a combination of agents, such as FOLFOX (leucovorin, 5FU, and oxaliplatin) or CapeOX (capecitabine and oxaliplatin). Moreover, chemotherapy with oxaliplatin, irinotecan, and 5FU/leucovorin and targeted therapy using epidermal growth factor receptor (EGFR) antibodies, such as cetuximab and panitumumab, are recommended for metastatic CRC [1-3]. However, many patients encounter cancer recurrence despite treatment. Because CRC recurrence is a major issue leading to poor survival rates, additional developments of novel therapeutics are necessary.

The literature indicates that cancer stem cells (CSCs) with higher self-renewal and pluripotency are responsible for tumor recurrence $[4,5]$ and that they are associated with metastatic CRC [6]. To the best of our knowledge, CSCs present high drug resistance [7,8] and antiapoptosis property $[9,10]$ to survive tumor chemotherapies. Furthermore, CSCs induce the activation of oncogenic pathways, such as MET, HER2, and Wnt, for escaping the targeted therapies [11-15]. Therefore, to develop an effective therapy targeting specific driver genes, investigating the molecular mechanism of CSCs is essential [16]. Leucine-rich repeat-containing G-protein-coupled receptor 5 (LGR5) is a specific marker of CRC stem cells [16, 17], which participates in and activates the Wnt signaling pathway [18-20]. Activation of the Wnt signaling pathway is known for maintaining the survival of CRC stem cells. LGR5 is crucial during embryogenesis as a marker of adult intestinal stem cells in the small intestines [21]. Crypt base columnar (CBC) cells with high expression of LGR5 located interspersedly among the differentiated Paneth cells are capable of dividing into functional cells in the intestinal tissues [22]. The colorectal stem cells are similar to CBC cells that express high LGR5 [23]. Thus, Wnt signaling activation maintains the survival of CRC cells.

Because CRCs overexpress EGFR in >90\% of clinical patients, the literature indicates that EGFR is involved in activating the JAK-STAT signaling pathway [24] for the survival of CSCs $[25,26]$. In fact, EGFR was reported to be responsible for maintaining the survival of cancer stem-like cells (CSLCs) in EGFR-positive cancers [27]. Therefore, EGFR downstream proteins, such as signal transducer and activator of transcription 3 (STAT3), play a crucial role in activating Wnt signaling in colon [28, 29] and ovarian [30] cancers. The crosstalk between STAT3 and the Wnt signaling pathway possibly occurs during miRNA-92 regulation [30]. STAT3 knockdown consequently reduces $\beta$-catenin expression in CRCs [29]. Furthermore, Wnt upregulates STAT3 for inhibiting cell differentiation in embryonic stem cells [31]. Additionally, suppression of STAT3 by its specific inhibitors $[32,33]$ leads to the prevention of Wnt signaling activation. The results demonstrate a crosstalk between STAT3 and the Wnt signaling pathway. In particular, $\beta$-catenin stabilized and activated by Wnt is predominantly detected in the invasive regions of colorectal carcinomas [34] where CSCs are responsible for tumor metastasis [35]. These pieces of evidence support that CSCs contribute to resistance against chemoradiotherapies, leading to tumor relapse [36]. Therefore, therapeutics that eradicate CSCs through targeting of specific driver genes are considered successful [6].

The aim of this study was to understand the potential molecular mechanisms of CSCs through RNAseq analysis followed by bioinformatics analysis for identifying the specific driver genes involved in the survival of cancer stem-like tumorspheres. This study also used in vitro therapeutic screening for identifying the targeted agents against EGFR-positive CRCs and their derived cancer stem-like tumorspheres. The CSLCs were derived from the colorectal HCT116 and HT29 cells through the addition of EGF, bFGF, insulin, and heparin in a serum-free cultured medium $[37,38]$. Therefore, the genes involved in the EGF-, FGF-, and insulin-mediated signal transduction pathways were priorly investigated through RNAseq analysis. Furthermore, we conducted the study to identify the inhibitory agents against the driver genes in the $\mathrm{CRC}$-derived tumorspheres and compare them with the results obtained from RNAseq and bioinformatics analyses.

\section{Methods \\ Cell culture and tumorsphere formation}

The colorectal HCT116 and HT29 cancer cells were gifted to us by the Institute of Nuclear Energy Research, Taiwan. They were free of mycoplasma. The HCT116 cells were cultured in McCoy's 5A medium, and the HT29 cells were cultured in Dulbecco's Modified Eagle's Medium with $10 \%$ fetal bovine serum and $1 \%$ penicillinstreptomycin. Tumorsphere formation and measurement were the same as described previously [37]. Plateletderived growth factor (PDGF)-AA was purchased from R\&D Systems, Inc. (Minneapolis, MN, USA).

\section{EGFR measurement through flow cytometry}

The obtained H520, HCT116, and HT29 cells were treated with $2 \mu \mathrm{g} / \mathrm{mL}$ of cetuximab-FITC for $2 \mathrm{~h}$ at $4{ }^{\circ} \mathrm{C}$. Cetuximab-FITC was created and validated as in a 
previous study [39]. Subsequently, the medium was removed and the cells were washed thrice with phosphate-buffered saline (PBS). The cells in PBS buffer were analyzed for EGFR expression using a FACSCalibur Flow Cytometer (BD Bioscience, San Jose, CA, USA). In this study, H520 was used as an EGFR-negative cell line.

\section{Quantitative polymerase chain reaction}

The procedure for mRNA extraction and complementary DNA preparation was the same as described previously [37]. Quantitative polymerase chain reaction (qPCR) was performed using the SYBR Green system (Applied Biosystems, Foster City, CA, USA) according to the manufacturer's instruction. The primers are presented in Table 1.

\section{RNAseq and bioinformatics analyses}

RNAseq analysis was performed to compare the differential levels of genes between the HCT116 cells and HCT116-derived tumorspheres using HiSeq 4000 with paired-end $150 \mathrm{bp}$ sequencing. The differential genes are shown in Additional file 1: Table S1. The genes that showed a $>1$-fold change $(\log 2)$ in expression in the HCT116-derived tumorspheres compared with the parental HCT116 cells were classified according to their molecular function using the PANTHER classification system (http://pantherdb.org/). The genes that showed > 3 -fold change $(\log 2)$ in expression in the HCT116-derived tumorspheres are presented in Table 2 . In addition, the genes classified using PANTHER that are associated with the EGF, FGF, and insulin pathways are presented in Table 3. Moreover, using the Kaplan-Meier plotter derived from the database PROGgeneV2 (http://watson.compbio.iupui.edu/chirayu/proggene/database/index.php), we identified the relationship between the overall survival rate and mRNA expression of the target genes. The protein--protein interaction was analyzed using NetworkAnalyst (http://www.networkanalyst.ca/), and pathway activations were selected and matched according to the KEGG database.

Table 1 Primers used in this study

\begin{tabular}{lll}
\hline Gene & Direction & Sequence (5' to 3') $^{\prime}$ \\
\hline LGR5 & Forward & CTCTTCCTCAAACCGTCTGC \\
& Reverse & GATCGGAGGCTAAGCAACTG \\
CD133 & Forward & CTATTCAGGATATACTCTCAGCATT \\
& Reverse & TTCTGTGGATGTAACTTCAGTG \\
PDGFA & Forward & ACGTCAGGAAGAAGCCAAAA \\
& Reverse & GGCTCATCCTCACCTCACAT \\
GAPDH & Forward & GAGTCAACGGATTGGTCGT \\
& Reverse & TTGATITGGAGGGATCTCG \\
\hline
\end{tabular}

Table 2 Genes that showed > 3-fold change $(\log 2)$ in expression in $\mathrm{HCT116-derived} \mathrm{tumorspheres}$

\begin{tabular}{|c|c|c|}
\hline Gene & fold chang $(\log 2)$ & qvalue \\
\hline$\overline{G B P 2}$ & 5.234 & $7.48 \mathrm{E}-05$ \\
\hline KRTAP2-3 & 4.9065 & $1.31 \mathrm{E}-36$ \\
\hline LUCAT1 & 4.8558 & $2.11 \mathrm{E}-10$ \\
\hline ITGAX & 4.66 & 4.87E-09 \\
\hline TMEM158 & 4.4984 & $3.71 \mathrm{E}-85$ \\
\hline CREB5 & 4.4312 & $5.02 \mathrm{E}-10$ \\
\hline EFEMP2 & 4.3996 & $1.17 \mathrm{E}-11$ \\
\hline ANKRD37 & 4.3836 & $6.70 \mathrm{E}-13$ \\
\hline ANGPTL4 & 4.3529 & $3.55 \mathrm{E}-103$ \\
\hline NDRG1 & 4.1854 & 0 \\
\hline IZUMO4 & 4.0022 & $1.24 \mathrm{E}-12$ \\
\hline PMEPA1 & 3.951 & 0.000462 \\
\hline LINC00202-1 & 3.9266 & 0.004557 \\
\hline$H L A-V$ & 3.9176 & 0.001176 \\
\hline CXCL8 & 3.8982 & $2.38 \mathrm{E}-20$ \\
\hline$K B-1460 A 1.5$ & 3.7413 & 0.000276 \\
\hline C1orf228 & 3.657 & 0.002742 \\
\hline IGFBP3 & 3.5875 & $3.18 \mathrm{E}-09$ \\
\hline HISTIHIC & 3.5841 & $1.94 \mathrm{E}-18$ \\
\hline PDGFA & 3.5807 & 1.44E-48 \\
\hline CITED4 & 3.5739 & 0.000183 \\
\hline CXCR4 & 3.5015 & 0.003701 \\
\hline STRC & 3.4502 & 0.004858 \\
\hline ISG20 & 3.3791 & 0.000322 \\
\hline HILPDA & 3.3395 & $5.75 E-36$ \\
\hline FAM83A & 3.339 & 0.00102 \\
\hline$A M Y 2 B$ & 3.2986 & 0.001327 \\
\hline ALDOC & 3.2823 & $1.74 \mathrm{E}-66$ \\
\hline$A M N$ & 3.2771 & 0.000458 \\
\hline YPEL3 & 3.2485 & 0.00012 \\
\hline SLPI & 3.2287 & 0.000305 \\
\hline RP11-102 K13.5 & 3.1821 & 0.000914 \\
\hline CYP4F12 & 3.177 & $1.68 \mathrm{E}-05$ \\
\hline AGPAT4 & 3.163 & 5.97E-24 \\
\hline CORIN & 3.111 & 0.00075 \\
\hline
\end{tabular}

\section{Cell viability}

The alarmarBlue assay was used according to the manufacturer's protocol to determine cell viability. Inhibitors against the HCT116 and HT29 cells were added to the cell culture and incubated for $48 \mathrm{~h}$. Cell viability was then measured using alarmarBlue. STAT3 inhibitors against HT29CSCs were added to the culture and incubated for 7 days because tumorsphere formation requires $>7$ days. 
Table 3 Upregulated genes associated with EGF, FGF, and insulin pathways in HCT116-derived tumorspheres (analyzed using PANTHER, http://pantherdb.org/)

\begin{tabular}{llll}
\hline Pathway & Gene & fold chang (log2) & qvalue \\
\hline EGF & RRAS & 1.9498 & $6.33 \mathrm{E}-25$ \\
& RAC2 & 1.1094 & $4.70 \mathrm{E}-07$ \\
& PIK3CD & 1.2025 & 0.002031 \\
& STAT2 & 1.1837 & $7.66 \mathrm{E}-06$ \\
& MAPK15 & 1.2498 & $5.45 \mathrm{E}-07$ \\
& PRKCG & 2.2982 & 0.0042873 \\
& SHC2 & 2.6131 & 0.0019177 \\
& STAT3 & 1.0825 & $1.18 \mathrm{E}-11$ \\
& PPP2R5B & 2.4064 & $1.95 \mathrm{E}-14$ \\
& SFN & 1.2086 & $2.92 \mathrm{E}-57$ \\
& AREG & 1.6495 & $2.22 \mathrm{E}-52$ \\
FGF & FGFR1 & 1.0804 & $3.25 \mathrm{E}-10$ \\
& RAC2 & 1.1094 & $4.70 \mathrm{E}-07$ \\
& PIK3CD & 1.2025 & 0.002031 \\
& PRKCG & 2.2982 & 0.0042873 \\
Insulin & PPP2R5B & 2.4064 & $1.95 \mathrm{E}-14$ \\
& SFN & 1.2086 & $2.92 \mathrm{E}-57$ \\
& IRS2 & 2.0046 & $1.06 \mathrm{E}-07$ \\
FOXO3 & 1.3511 & $9.38 \mathrm{E}-12$ \\
& PDK1 & 1.4964 & 0.00013109 \\
& PIK3CD & 1.2025 & 0.002031 \\
\hline
\end{tabular}

\section{Western blots}

Western blotting was conducted as previously described [37]. The specific antibodies against $\beta$-catenin, STAT3, pSTAT3 (Y705), and GAPDH were purchased from Cell Signaling (Danvers, MA, USA).

\section{Gene knockdown}

STAT3 was knocked down using a short-hairpin RNA (shRNA)-expression lentivirus system containing the specific shRNA target sequences (1) GCAAAGAAT CACATGCCACTT for HT29shSTAT3\#1 and (2) GCAC AATCTACGAAGAATCAA for HT29shSTAT3\#2 in the vector pLKO.1-puro that was generated in $293 \mathrm{~T}$ cells. The procedure was the same as that in our previous study [37].

\section{Animal}

Male NOD/SCID mice were purchased from BioLASCO Taiwan Co., Ltd., Taiwan. The 5-week-old mice were housed in a $12 \mathrm{~h}$-light cycle at $22{ }^{\circ} \mathrm{C}$. The animal studies were approved by the institutive ethical review committee in Mackay Memorial Hospital, Taiwan, which followed the $\mathrm{NIH}$ guidelines on the care and welfare of laboratory animals. Tumor xenografts were established by injecting
$2 \times 10^{6}$ HT29 $(n=3)$ or HT29shSTAT3\#2 cells $(n=3)$, into the subcutaneous legs of mice of 5 weeks-old. The tumor sizes were measured using a digital caliper and recorded on day 14, 17, 19, 21 and 24 . Tumor volume was recorded and calculated using the formula: $0.52 \mathrm{x}$ width ${ }^{2} \mathrm{x}$ length, herein the width represents the smaller tumor diameter.

\section{Statistical analysis}

Statistical analyses were performed using GraphPad Prism V5.01 (GraphPad Software, Inc., CA, USA). All analytical data with more than two groups were evaluated using analysis of variance, followed by post hoc analysis with Bonferroni's test. Student's t-test was used to compare two groups. Additionally, $p<0.05$ was considered an acceptable statistically significant difference.

\section{Results}

Formation of cancer stem-like tumorspheres from selective EGFR-positive CRCs

In this study, our first aim was to investigate the molecular mechanism of CSLCs derived from selective CRC cells that overexpressed EGFR, including HCT116 and HT29. The observed EGFR expression was validated through flow cytometry and compared with lung H520 cancer cells (Fig. 1a and b), which are EGFR-negative [37]. To analyze the CRC stem-like cells, four growth factors, namely EGF, bFGF, insulin, and heparin, were added to the HCT116 and HT29 cells and subsequently incubated. The culture methodology is described in the Materials and methods section. Both the HCT116 and HT29 cells formed tumorspheres within 7 days of incubation, reaching a size of $>100 \mu \mathrm{m}$ (Fig. 1c and d). Both HCT116CSC and HT29CSC expressed higher LGR5 levels without an increase in CD133 (Fig. 1e and f). Because LGR5 is a marker of CSCs, the CSC-associated genes were proposed to be upregulated in the tumorspheres; therefore, these tumorspheres were used as the study models for investigating the molecular mechanism of CSCs.

\section{Activation of STAT3 in HCT116-derived tumorspheres}

We investigated the major driver genes in the formation and survival of CRC stem-like cells by conducting RNAseq analysis. The differential genes between the HCT116-derived tumorspheres and parental HCT116 cells were identified. In total, 688 genes were increased and 1788 genes were decreased in the HCT116-derived tumorspheres compared with parental HCT116 cells (Fig. 2a). According to the statistical q value $(p<0.005$ with $\log 2$ fold change >1), 654 and 840 genes were significantly upregulated and downregulated, respectively, in the HCT116-derived tumorspheres (Fig. 2b and Additional file 1: Table S1 present all the differential 


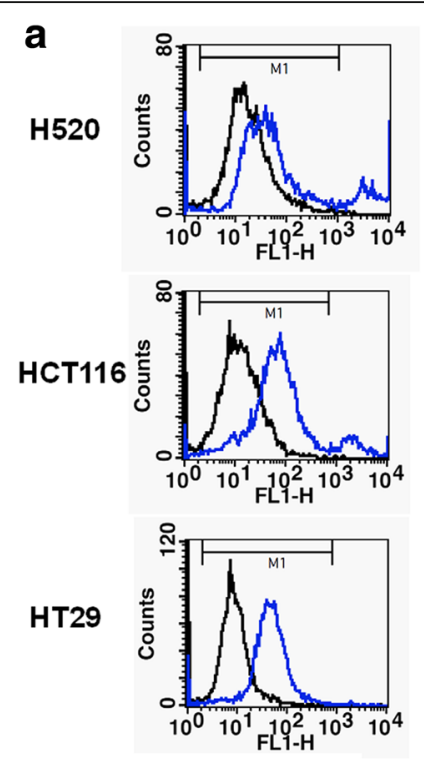

b

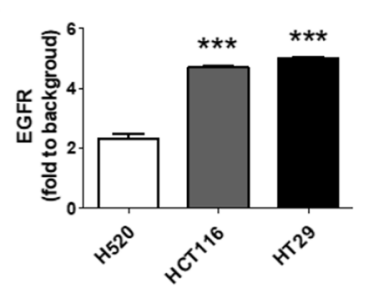

C

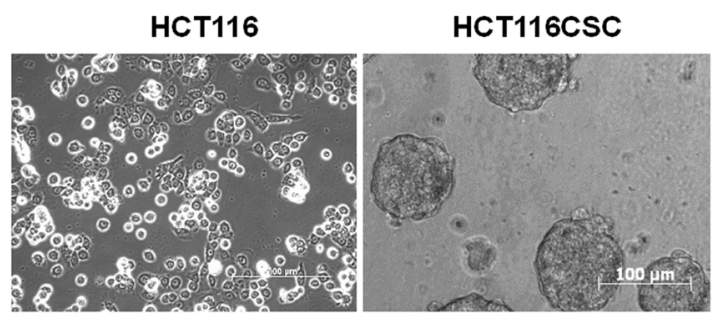

d

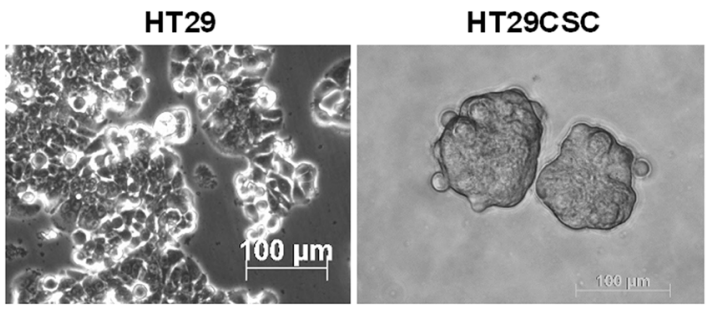

e

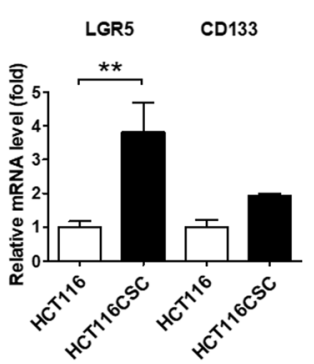

f

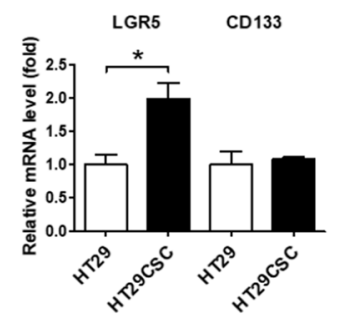

Fig. 1 EGFR-positive CRC-derived tumorspheres mimicking CSCS as study models. (a) EGFR-positive CRC cells HCT116 and HT29 were selected. These cancer cells were validated for their EGFR expression through flow cytometry and compared with H520 cells, which are EGFR-negative. (b) Quantification of EGFR through fluoresce intensity revealed higher EGFR expression in HCT116 and HT29 cells compared with H520 cells. Therefore, HCT116 and HT29 were used as EGFR-positive models in this study. The tumorspheres derived from (c) HCT116 (HCT116CSC) and (d) HT29 (HT29CSC) were cultured in low-attachment six-well plates with serum-free medium containing $20 \mathrm{ng} / \mathrm{mL}$ of EGF, $20 \mathrm{ng} / \mathrm{mL}$ of fibroblast growth factor, $5 \mu \mathrm{g} / \mathrm{mL}$ of bovine insulin, and $4 \mu \mathrm{g} / \mathrm{mL}$ of heparin for 7 days to form tumorspheres measuring approximately $100 \mu \mathrm{m}$ in diameter. (e and $\mathbf{f}$ ) qPCR revealed higher LGR5 expression in HCT116CSC and HT29CSC than in their respective parental cells; LGR5 is a known gastrointestinal stem cell marker. However, another stem cell marker CD133 was not significantly affected. Scale bar: $100 \mu \mathrm{m} .{ }^{*} p<0.05 .{ }^{* *} p<0.01$. ${ }^{* * *} p<0.001$

genes). Genes showing a $>3$-fold change (log2) in expression are listed in Table 2. Genes showing a $>1$-fold change $(\log 2)$ in expression in the HCT116-derived tumorspheres were classified according to their molecular function by using the PANTHER classification system (Additional file 2: Figure S1). Among the significant genes, we were interested in the genes involved in the EGF, FGF, and insulin pathways because the tumorspheres were cultured using these growth factors. The EGF-, FGF-, and insulin-pathway-associated genes classified by PANTHER are shown in Table 3.

To analyze the interaction among the genes that showed a $>3$-fold change $(\log 2)$ in expression (Table 2 ) or genes associated with the EGF, FGF, and insulin pathways (Table 3), NetworkAnalyst was used. In NetworkAnalyst analysis, KEGG associated with cancer pathways showed that PDGFA was indicative (Fig. 2c) and STAT3 may be involved in activating the formation of cancer stem-like tumorspheres (Fig. 2c). The inceased PDGFA levels were consequently validated in both HCT116CSC and HT29CSC through qPCR (Additional file 3: Figure S2). Furthermore, the HCT116 cells were treated with EGF, bFGF, and insulin individually to determine the growth factor playing a crucial role in inducing PDGFA; EGF was found to significantly induce the expressin of PDGFA in the HCT116 cells (Fig. 2d).

To demonstrate that PDGFA was significant in CRCs, we used the Kaplan-Meier plotter according to the PROGgeneV2 database. We found that higher levels of PDGFA were associated with poor survival in four cohorts of the clinical trial datasets GSE28814, GSE28722, GSE41258, and GSE29621 (Additional file 4: Figure S3) among the 12 available datasets. Moreover, $20 \mathrm{ng} / \mathrm{mL}$ of 
a

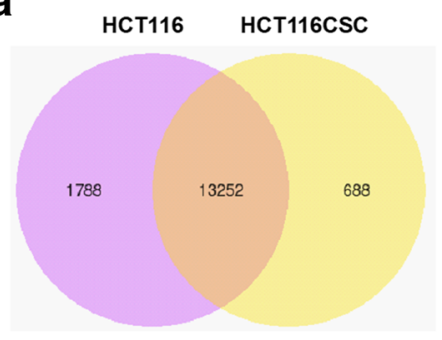

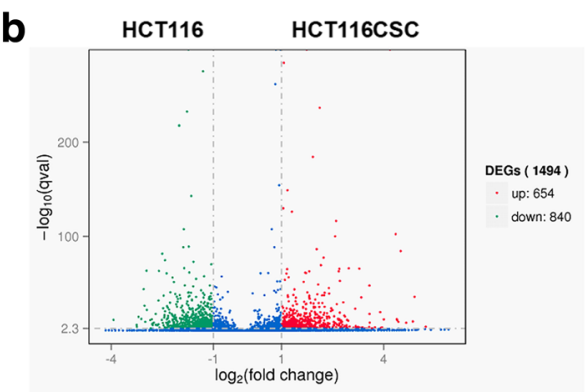

C

Pathways in cancer / NetworkAnalyst

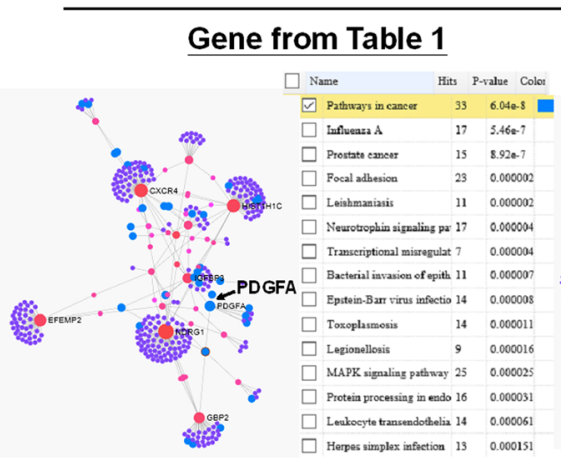

d

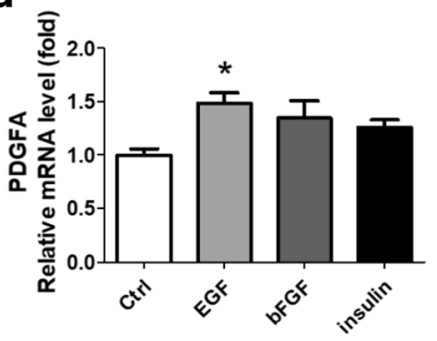

e

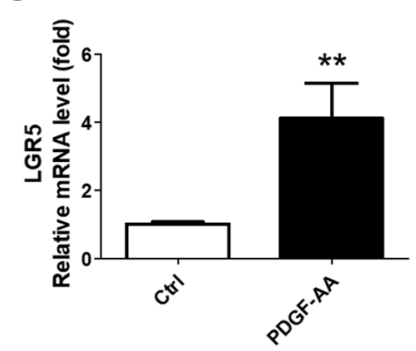

Gene from Table 2

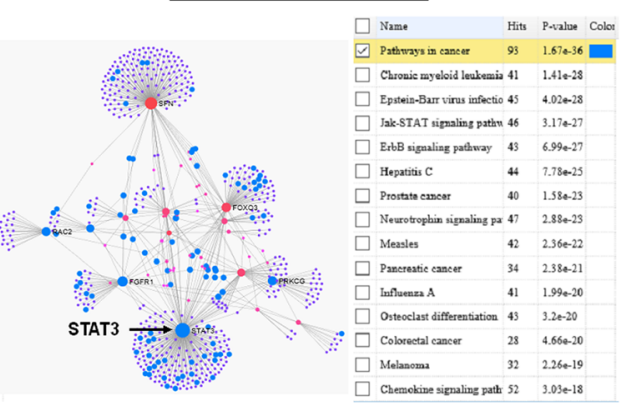

f

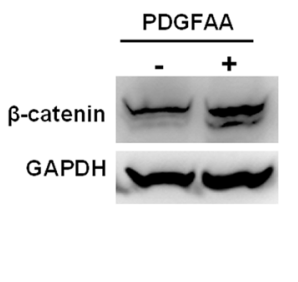

Fig. 2 Gene expression profile of HCT116-derived tumorspheres investigated through RNAseq analysis indicated that PDGFA and STAT3 were significant. (a) In total, 688 genes increased and 1788 genes decreased in the HCT116-derived tumorspheres compared with the parental HCT116 cells. (b) Numbers of significant genes upregulated and downregulated in the HCT116-derived tumorspheres according to $q$ value $(p<0.005$ with $>1$ fold change by log2) were 654 and 840, respectively. (c) Upregulated genes in the HCT116-derived tumorspheres were classified according to their molecular functions using PANTHER software (http://pantherdb.org/) and shown in Additional file 2: Figure S1. Upregulated genes with a > 3-fold change (log2) (Table 1) and genes associated with EGF, FGF, and insulin (Table 2) were analyzed using NetworkAnalyst (http://www.networkanalyst.ca/) for identifying the relevant signaling pathways based on the KEGG database. Genes associated with the cancer pathway are indicated by blue spots, revealing the overexpression of PDGFA and STAT3, indicated by arrows. (d) Through GPCR, the growth factor inducing PDGFA levels was identified. Thus, $20 \mathrm{ng} / \mathrm{mL}$ of EGF significantly induced PDGFA levels. (e) PDGF-AA was consequently demonstrated to be capable of inducing $L$ GR5 levels and (f) leading to $\beta$-catenin expression. ${ }^{*} p<0.05 .{ }^{* *} p<0.01$

PDGF-AA added to the HCT116 cells was capable of inducing the expression of not only LGR5 (Fig. 2e) but also $\beta$-catenin (Fig. 2f), a protein stabilized by Wnt, indicating that PDGFA overexpression in the cancer stem-like tumorspheres played a major role in inducing the activation of the Wnt signaling pathway. Furthermore, the Wnt-associated genes classfied using PANTHER are presented in Additional file 5: Table S2, revealing that Wnt7A, Wnt7B, Wnt9A, and others were upregulated in the tumorspheres, which indicates the activation of the Wnt signaling pathway.
Therapeutic screening for identifying the potential agents against EGFR-positive CRCs

Because CSCs contribute to drug resistance and tumor recurrence, the Compound Screening Library (MedChemExpress, NJ, USA) targeting stem cells was used for identifying the potential therapeutics against EGFR-positive CRCs. A panel containing 172 compounds was added to HCT116 and HT29 cells separately at a dose of $1 \mu \mathrm{M}$ and incubated for $48 \mathrm{~h}$ (Fig. 3a). The compounds that reduced the cell viabilities of both HCT116 and HT2 29 by $60 \%$ are marked in red. In total, 8 of the 172 


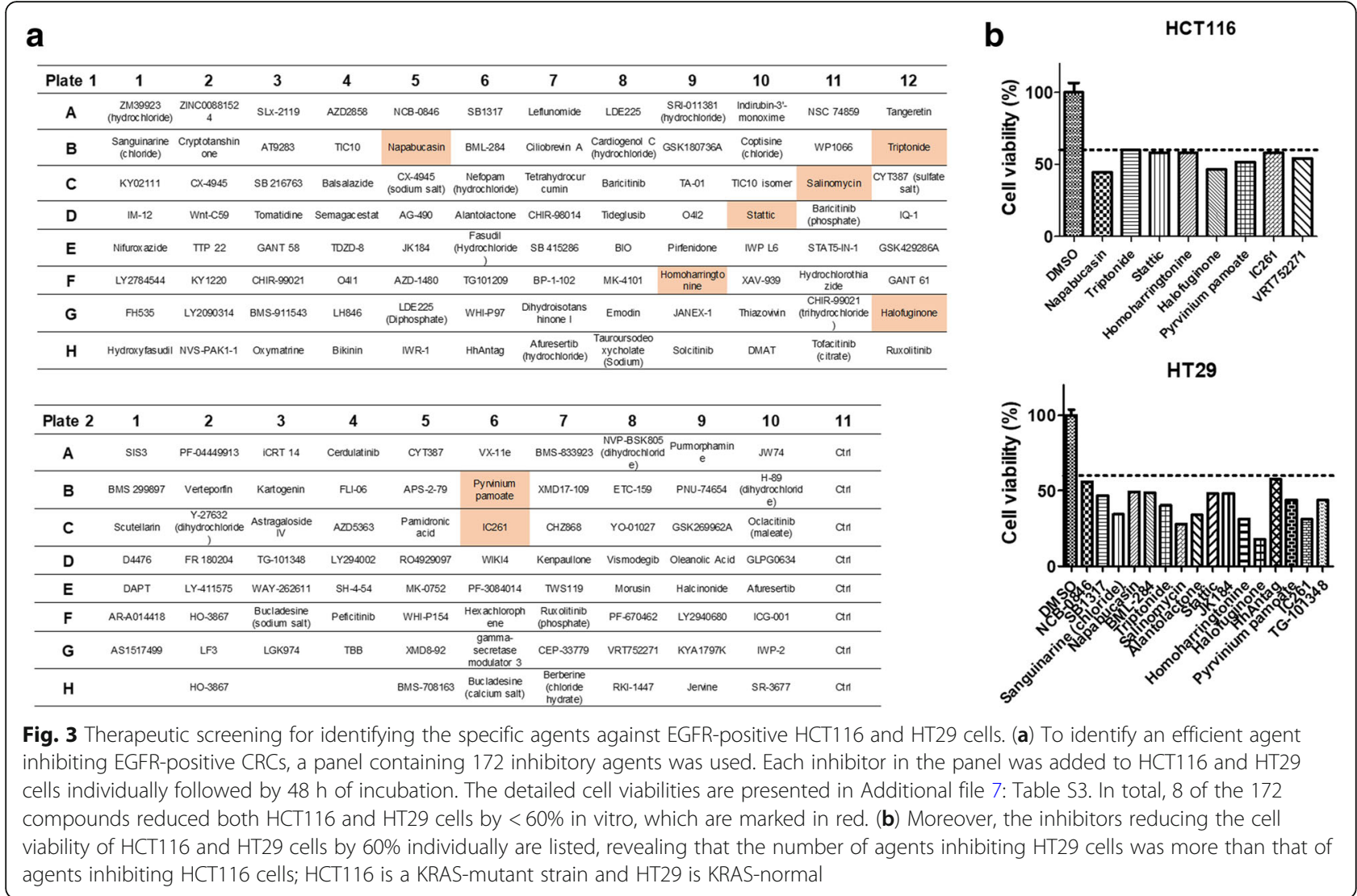

compounds significantly reduced the viabilities of the two CRCs (Fig. 3a); the compounds targeted genes such as STAT3 (napabucasin, homoharringtonine, and stattic), Wnt (pyrvinium pamoate, triptonide, salinomycin, IC261), and transforming growth factor beta (TGF $\beta$ )/smad (halofuginone). The compound structures are shown in Additional file 6: Figure S4. The individual therapeutics against HCT116 and HT29 are illustrated in Fig. 3b, and the detailed experimental results are presented in Additional file 7: Table S3. The results indicated that the STAT3, Wnt, and TGF $\beta$ /smad signaling pathways were involved in the survival of the selective EGFR-positive CRCs.

\section{Targeting STAT3 inhibited the formation and survival of CRC stem-like tumorspheres}

To investigate and validate that STAT3 was involved in the formation and survival of CRC stem-like tumorspheres, compounds targeting STAT3 were used. Napabucasin, homoharringtonine, and stattic, which significantly reduced the cell viabilities of the HCT116 and HT29 cells, were measured for their inhibitory concentrations (IC50 values) against the HCT116 and HT29 cells in a dose-dependent manner from 0 to $2 \mu \mathrm{M}$ (Fig. 4a). According to the IC50 results (Fig. 4b), napabucasin specifically inhibited HCT116 with an IC50 value of $1.02 \mu \mathrm{M}$ and homoharringtonine specifically inhibited HT29 with an IC50 value of $0.89 \mu \mathrm{M}$. The results were consistent with the therapeutic screening results shown in Fig. 3b. Furthermore, napabucasin and homoharringtonine were added and investigated for their activities against the formation of HT29-derived tumorspheres. The results revealed that both napabucasin and homoharringtonine inhibited the formation of HT29-derived tumorspheres at a dose of 0.5 and $0.1 \mu \mathrm{M}$, respectively (Fig. 4c), including reducing the diameter (size, $\mu \mathrm{m})$ and cell viability. We speculate that the lower inhibitory concentration against HT29-derived tumorspheres than against the parental HT29 cells was because the incubation time exceeded 7 days. Because the tumorspheres were cultured with the addition of the growth factors EGF, bFGF, and insulin, they were analyzed using Western blot analysis to identify the growth factor responsible for activating STAT3. EGF significantly induced STAT3 phosphorylation and elevated the mRNA levels of LGR5 in the HT29 cells (Fig. 4d), implying that addition of EGF activated STAT3 in the cancer stem-like tumorspheres, thus contributing to the formation and survival of EGFR-positive CRC stem-like tumorspheres. 


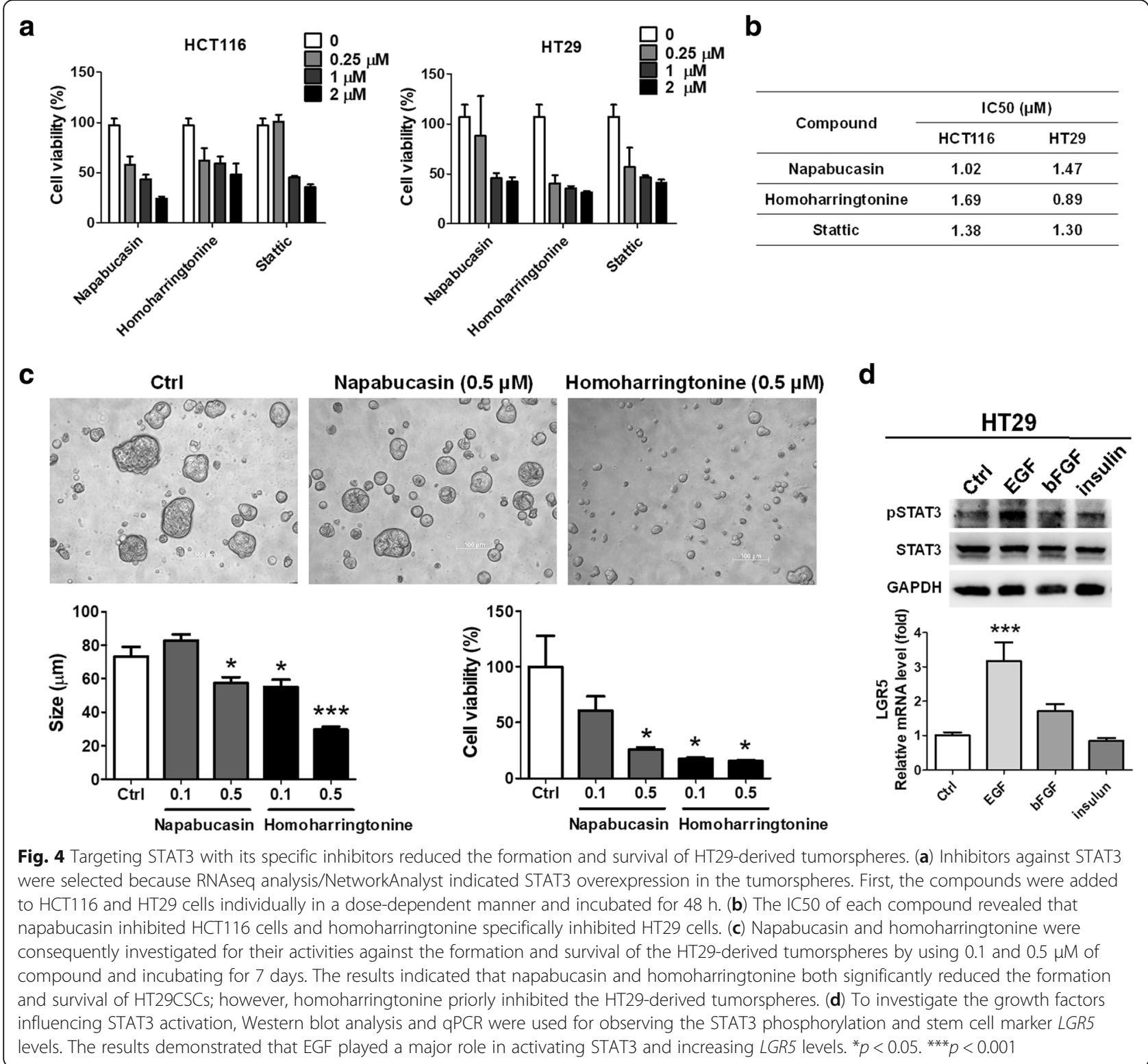

STAT3 knockdown reduced expression of PDGFA and survival of HT29 cells and their derived tumorspheres through impairment of EGF-induced Wnt activation

To validate that STAT3 was involved in the formation and survival of the cancer stem-like tumorspheres, STAT3 was knocked down (Fig. 5a). However, STAT3 knockdown did not sufficiently inhibit the $\beta$-catenin expression (data not shown), a protein stabilized in Wnt signaling activation. An in-depth investigation revealed that the mRNA levels of PDGFA decreased in the HT29shSTAT3 cells (Fig. 5a). Furthermore, STAT3 knockdown significantly reduced the cell viability of the HT29 cells (Fig. 5b) and tumor growth in tumor xenografts (Fig. 5c). Thus, STAT3 determined cell survival in EGFR-positive colorectal tumors. Moreover, STAT3 knockdown inhibited the formation and survival of tumorspheres (Fig. 5d). To investigate whether Wnt signaling activation was regulated by the EGF-STAT3 pathway, $20 \mathrm{ng} / \mathrm{mL}$ of EGF was added to the HT29 and HT29shSTAT3 cells individually. The results indicated that EGF induced STAT3 phosphorylation, leading to $\beta$-catenin overexpression, which had no effect on the HT29shSTAT3 cells (Fig. 5e). Consequently, the HT29 cells were treated with the STAT3 inhibitors to clarify the mechanism of homoharringtonine, which significantly inhibited the formation and survival of tumorspheres (Fig. 4c). Only homoharringtonine remarkably inhibited STAT3 expression when the cells were treated with $1 \mu \mathrm{M}$ of compound for $48 \mathrm{~h}$ (Fig. 5f), resulting in reduced EGF-mediated $\beta$-catenin expression. Thus, 


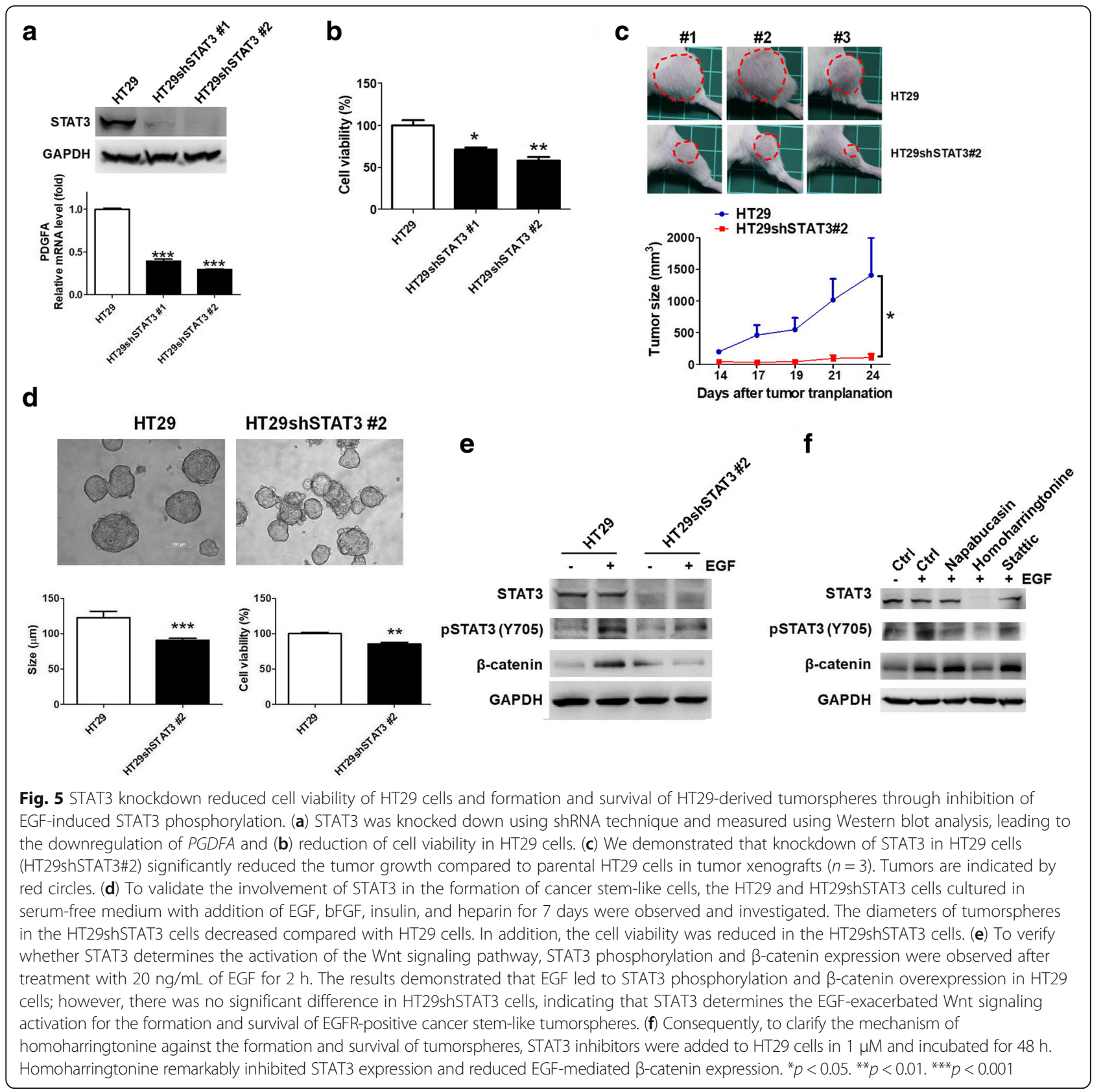

STAT3 is capable of exacerbating $\beta$-catenin and inducing PDGFA to promote Wnt signaling for maintaining the formation and survival of cancer stem-like tumorspheres in selective EGFR-positive CRCs.

\section{Discussion}

This study conducted gene profiling analyses of colorectal HCT116CSCs through RNAseq analysis and determined that STAT3 played a crucial role in maintaining the CRC-stem-like tumorspheres. Furthermore, PDGFA overexpressed in the CSCs, specifically induced through the EGF-STAT3 pathway, increased the levels of LGR5, a marker of CRC stem cells, participating in the Wnt signaling pathway. Thus, EGF contributed to the survival of CSCs by STAT3 to activate the PDGFA-mediated Wnt signaling pathway in the selective EGFR-positive CRCs.

HCT116 has been demonstrated as an EGFR-overexpressed cell line [40, 41], possessing high stemness [42]. Because we previously demonstrated that EGFR may contribute to the formation of cancer stem-like tumorspheres in lung cancers [37], we assumed that EGFR may also contribute to cancer stemness in CRCs. To test the hypothesis, we selected EGFR-positive HCT116 and 
HT29 cell lines as study models for investigating the molecular mechanism of CSCs that were considered responsible for tumor metastasis and recurrence. We found that STAT3 activation (phosphorylation) and LGR5 mRNA levels were individually induced by EGF, revealing that EGFR is capable of exacerbating cancer stemness properties; this finding is consistent with that of a previous study [43]. We speculate that EGF induces EGFR phosphorylation [37], leading to signaling cascades and thus resulting in STAT3-mediated PDGFA overexpression and Wnt signaling activation. Because LGR5 expression was induced by both EGF and PDGF-AA, we concluded that overexpressed PDGFA induced by EGF seems to be able to exacerbate CRC stemness.

Therapeutic screening using a panel containing 172 inhibitors against stem cells revealed that targeting the STAT3, Wnt, and TGF $\beta /$ smad pathways significantly reduced selective EGFR-positive CRC survival. Thus, inhibition of STAT3 expression using homoharringtonine and knockdown techniques validated that STAT3 was a driver gene contributing to the formation and survival of the CSLCs derived from the EGFR-positive CRCs. However, formation of cancer stem-like tumorspheres was still observed in the HT29shSTAT3 cells, although STAT3 expression was downregulated, as observed using Western blot analysis, indicating that blockade of STAT3 may be insufficient to completely eradicate CRCs. According to the therapeutic screening results, inhibitors targeting Wnt and TGF $\beta /$ smad should be of interest for future investigations of their activities against EGFR-positive CRCs. Anti-EGFR therapies, such as cetuximab and panitumumab, are suggested to cure patients with CRCs [44]. However, mutation of genes such as KRAS, NRAS, and BRAF in CRCs promotes cell proliferation and evokes drug resistance against anti-EGFR therapies [45]. Downstream signaling targets have been suggested for reducing drug resistance and preventing tumor recurrence. This study not only established the well-known STAT3 as a potential therapeutic target but also identified a specific anti-STAT3 agent, homoharringtonine, against EGFR-positive CRCs. Homoharringtonine, in particular, inhibited the formation and survival of the CSLCs through STAT3 expression downregulation, which resulted in a remarkable downregulation of EGF-mediated $\beta$-catenin. Homoharringtonine prevents the initial elongation step of protein synthesis [46]. However, Cao and collegues demonstrated that homoharringtonine also reduces pSTAT3 on Y705 in lung cancer cells [47]. We found that STAT3 knockdown reduced EGF-mediated $\beta$-catenin expression in this study, and this result is consistent with that of a previous study demonstrating that STAT3 mediated $\beta$-catenin in nine CRC cell lines [29]. Therefore, homoharringtonine probably reduced EGF-mediated $\beta$-catenin through primarily inhibiting STAT3 expression.

We also found that PDGFA overexpression was regulated by the EGF-STAT3 pathway, implying that the PDGF-AA binding receptor PDGFR $\alpha \alpha$ served as another potential driver activating Wnt signaling, which could be a potential target against tumor recurrence [48]. A previous study indicated that PDGF-AA and PDGF-BB can express PDGFRs through an autocrine mechanism [49]. Therefore, although STAT3 was knocked down, causing downregulation of PDGFA and lower cell viability in the HT29 cells in this study (Fig. 5a, b, c), it was insufficient to inhibit $\beta$-catenin and completely block tumorsphere formation. Hence, we speculate that PDGFA is probably also induced by either bFGF or insulin (Fig. 2d) to lead to the autocrine mechanism of PDGF-PDGFR activation because PDGFR plays a crucial role in triggering and maintaining cancer stemness [50].

\section{Conclusion}

Comparing whole gene expression profiles using PANTHER and NetworkAnalyst in combination with RNAseq, we determined the role of STAT3 activation in CRC-derived cancer stem-like tumorspheres. Additionally, we validated that PDGFA increased in the tumorspheres through the EGF-STAT3 pathway, leading to the induction of $L G R 5$ and Wnt signaling. The therapeutic screening and knockdown experiment confirmed the observation, indicating that STAT3 targeting is a potential treatment strategy against EGFR-positive CRCs. This study not only elucidated the molecular mechanism of CSLCs but also provided responsible targeted inhibitors such as homoharringtonine against selective EGFR-positive CRCs.

\section{Additional files}

Additional file 1: Table S1. All the differential genes analyzed using RNAseq shown in the attached excel file. (XLS $902 \mathrm{~kb}$ )

Additional file 2: Figure S1. Differentially upregulated genes were classified using PANTHER, showing the gene number in distinguished functions. (TIF $161 \mathrm{~kb}$ )

Additional file 3: Figure S2. PDGFA levels were validated to be upregulated in the tumorspheres compared with their parental cells derived from EGFR-positive HCT116 and HT29 cells. (TIF 47 kb)

Additional file 4: Figure S3. Higher levels of PDGFA are associated with overall survival in patients with CRC in the database PROGgeneV2. (TIF 226 kb)

Additional file 5: Table S2. Upregulated genes associated with the Wnt signaling pathway in HCT116-derived tumorspheres (analyzed using PANTHER, http://pantherdb.org/). (DOCX 14 kb)

Additional file 6: Figure S4. Chemical structures of the eight compounds that significantly reduced the cell viabilities of both HCT116 and HT29 cells by $60 \%$. (TIF $287 \mathrm{~kb}$ )

Additional file 7: Table S3. Detailed measurement of cell viability in therapeutic screening against HCT116 and HT29 cells. (DOCX 632 kb) 


\section{Acknowledgments}

We thank Ling Huang for her technical support. This manuscript was edited by Wallace Academic Editing.

\section{Funding}

This study was supported by grants from the Ministry of Science and Technology of Taiwan (MOST 106-2320-B195-003, MOST 106-2320-B-038 -048). This work was supported by Health and welfare surcharge of tobacco products grant MOHW107-TDU-B-212-114014, Cheng Hsin General Hospital (CHGH 106-06), and Mackay Memorial Hospital (MMH-CT-10605 and MMH-106-61).

\section{Availability of data and materials}

Using the Kaplan-Meier plotter according to the database PROGgeneV2 (http://watson.compbio.iupui.edu/chirayu/proggene/database/index.php), we identified the relationship between the overall survival rate of colorectal cancer and mRNA expression of the target genes.

\section{Authors' contributions}

C-CCheng and Y-FC conceptualized and designed the study. C-CCheng and A-SH developed the methodology. C-CCheng and $\mathrm{K}-\mathrm{HL}$ collected the data. C-CCheng, JC, and B-LY analyzed and interpreted the data (e.g., statistical analysis, biostatistics, and computational analysis). C-CCheng, P-NL, K-HL, Y$\mathrm{FC}$, and $\mathrm{C}-\mathrm{CC}$ hang contributed toward writing, reviewing, and revising the manuscript. Y-WS, H-CL, CG-SC, and Y-CC provided administrative, technical, or material support (i.e., organizing data and constructing databases). Y-FC and C-CChang supervised the study. All authors read and approved the final manuscript.

\section{Ethics approval and consent to participate}

Not applicable.

\section{Consent for publication}

Not applicable.

\section{Competing interests}

The authors declare that they have no competing interests.

\section{Publisher's Note}

Springer Nature remains neutral with regard to jurisdictional claims in published maps and institutional affiliations.

\section{Author details \\ 'Division of Hematology and Oncology, Department of Internal Medicine, Mackay Memorial Hospital, Taipei, Taiwan. ${ }^{2}$ Laboratory of Good Clinical Research Center, Department of Medical Research, Mackay Memorial Hospital, Tamsui District, New Taipei City, Taiwan. ${ }^{3}$ Division of Gastroenterology, Cheng Hsin General Hospital, Taipei, Taiwan. ${ }^{4}$ Graduate Institute of Medical Sciences, School of Medicine, College of Medicine, Taipei Medical University, Taipei, Taiwan. ${ }^{5}$ Division of Gastroenterology and Hepatology, Department of Internal Medicine, Taipei Medical University Hospital, Taipei, Taiwan. 'Division of Gastroenterology and Hepatology, Department of Internal Medicine, School of Medicine, College of Medicine, Taipei Medical University, Taipei, Taiwan. ${ }^{7}$ Department of Medicine, MacKay Medical College, New Taipei City, Taiwan.}

\section{Received: 6 February 2018 Accepted: 26 June 2018}

\section{Published online: 02 August 2018}

\section{References}

1. New Guideline on Managing Colorectal Cancer. Cancer discovery. 2017;7(4): OF2.

2. Parseghian $C M$, Parikh NU, Wu JY, Jiang ZQ, Henderson L, Tian F, Pastor B, Ychou M, Raghav K, Dasari A, Fogelman DR, Katsiampoura AD, Menter DG, Wolff RA, Eng C, Overman MJ, Thierry AR, Gallick GE, Kopetz S. Dual inhibition of EGFR and c-Src by Cetuximab and Dasatinib combined with FOLFOX chemotherapy in patients with metastatic colorectal Cancer. Clin Cancer Res. 2017;23(15):4146-54.

3. Cascinu S, Rosati G, Nasti G, Lonardi S, Zaniboni A, Marchetti P, Leone F, Bilancia D, laffaioli RV, Zagonel V, Giordano M, Corsi DC, Ferrau F, Labianca R, Ronzoni M, Scartozzi M, Galli F. Investigators G. Treatment sequence with either irinotecan/cetuximab followed by FOLFOX-4 or the reverse strategy in metastatic colorectal cancer patients progressing after first-line FOLFIRI/ bevacizumab: an Italian Group for the Study of gastrointestinal Cancer phase III, randomised trial comparing two sequences of therapy in colorectal metastatic patients. Eur J Cancer. 2017;83:106-15.

4. Zhao Y, Peng J, Zhang E, Jiang N, Li J, Zhang Q, Zhang X, Niu Y. CD133 expression may be useful as a prognostic indicator in colorectal cancer, a tool for optimizing therapy and supportive evidence for the cancer stem cell hypothesis: a meta-analysis. Oncotarget. 2016;7(9):10023-36.

5. Choi JE, Bae JS, Kang MJ, Chung MJ, Jang KY, Park HS, Moon WS. Expression of epithelial-mesenchymal transition and cancer stem cell markers in colorectal adenocarcinoma: Clinicopathological significance. Oncol Rep. 2017:38(3):1695-705.

6. Massard C, Deutsch E, Soria JC. Tumour stem cell-targeted treatment: elimination or differentiation. Ann Oncol. 2006;17(11):1620-4.

7. Grillet F, Bayet E, Villeronce O, Zappia L, Lagerqvist EL, Lunke S, CharafeJauffret E, Pham K, Molck C, Rolland N, Bourgaux JF, Prudhomme M, Philippe C, Bravo S, Boyer JC, Canterel-Thouennon L, Taylor GR, Hsu A, Pascussi JM, Hollande F, Pannequin J. Circulating tumour cells from patients with colorectal cancer have cancer stem cell hallmarks in ex vivo culture. Gut. 2017;66(10):1802-10.

8. Dallas NA, Xia L, Fan F, Gray MJ, Gaur P, van Buren G 2nd, Samuel S, Kim MP, Lim SJ, Ellis LM. Chemoresistant colorectal cancer cells, the cancer stem cell phenotype, and increased sensitivity to insulin-like growth factor-I receptor inhibition. Cancer Res. 2009;69(5):1951-7.

9. Catalano V, Gaggianesi M, Spina V, lovino F, Dieli F, Stassi G, Todaro M. Colorectal cancer stem cells and cell death. Cancers. 2011;3(2):1929-46.

10. Piggott L, Omidvar N, Marti Perez S, French R, Eberl M, Clarkson RW. Suppression of apoptosis inhibitor C-FLIP selectively eliminates breast cancer stem cell activity in response to the anti-cancer agent, TRAlL. Breast cancer research : BCR. 2011;13(5):R88.

11. Miekus $\mathrm{K}$. The met tyrosine kinase receptor as a therapeutic target and a potential cancer stem cell factor responsible for therapy resistance (review). Oncol Rep. 2017;37(2):647-56.

12. Jarde T, Kass L, Staples M, Lescesen H, Carne P, Oliva K, McMurrick PJ, Abud HE. ERBB3 positively correlates with intestinal stem cell markers but marks a distinct non proliferative cell population in colorectal Cancer. PLoS One. 2015;10(9):e0138336.

13. Roesler R, Cornelio DB, Abujamra AL, Schwartsmann G. HER2 as a cancer stem-cell target. The Lancet Oncology. 2010;11(3):225-6.

14. Chen JF, Luo X, Xiang LS, Li HT, Zha L, Li N, He JM, Xie GF, Xie X, Liang HJ. EZH2 promotes colorectal cancer stem-like cell expansion by activating p21cip1-Wnt/beta-catenin signaling. Oncotarget. 2016;7(27):41540-58.

15. Ordonez-Moran P, Dafflon C, Imajo M, Nishida E, Huelsken J. HOXA5 counteracts stem cell traits by inhibiting Wnt signaling in colorectal Cancer. Cancer Cell. 2015;28(6):815-29.

16. Medema JP. Targeting the colorectal Cancer stem cell. N Engl J Med. 2017;377(9):888-90

17. Kleist $B, X u L, L i G$, Kersten C. Expression of the adult intestinal stem cell marker Lgr5 in the metastatic cascade of colorectal cancer. Int J Clin Exp Pathol. 2011;4(4):327-35.

18. de Lau W, Barker N, Low TY, Koo BK, Li VS, Teunissen H, Kujala P, Haegebarth A, Peters PJ, van de Wetering M, Stange DE, van Es JE, Guardavaccaro D, Schasfoort RB, Mohri Y, Nishimori K, Mohammed S, Heck AJ, Clevers H. Lgr5 homologues associate with Wnt receptors and mediate R-spondin signalling. Nature. 2011;476(7360):293-7.

19. Lin YU, Wu T, Yao Q, Zi S, Cui L, Yang M, Li J. LGR5 promotes the proliferation of colorectal cancer cells via the Wnt/beta-catenin signaling pathway. Oncol Lett. 2015;9(6):2859-63.

20. Vieira GC, Chockalingam S, Melegh Z, Greenhough A, Malik S, Szemes M, Park JH, Kaidi A, Zhou L, Catchpoole D, Morgan R, Bates DO, Gabb PD, Malik K. LGR5 regulates pro-survival MEK/ERK and proliferative Wnt/beta-catenin signalling in neuroblastoma. Oncotarget. 2015;6(37):40053-67.

21. Nigmatullina L, Norkin M, Dzama MM, Messner B, Sayols S, Soshnikova N. Id2 controls specification of Lgr5+ intestinal stem cell progenitors during gut development. EMBO J. 2017;36(7):869-85.

22. Anderson EC, Hessman C, Levin TG, Monroe MM, Wong MH. The role of colorectal cancer stem cells in metastatic disease and therapeutic response. Cancers. 2011;3(1):319-39. 
23. van de Wetering $M$, Sancho E, Verweij C, de Lau W, Oving I, Hurlstone A, van der Horn K, Batlle E, Coudreuse D, Haramis AP, Tjon-Pon-Fong M, Moerer P, van den Born M, Soete G, Pals S, Eilers M, Medema R, Clevers H. The beta-catenin/TCF-4 complex imposes a crypt progenitor phenotype on colorectal cancer cells. Cell. 2002;111(2):241-50.

24. Hambek M, Baghi M, Strebhardt K, May A, Adunka O, Gstottner W, Knecht R. STAT 3 activation in head and neck squamous cell carcinomas is controlled by the EGFR. Anticancer Res. 2004;24(6):3881-6.

25. Lin L, Jou D, Wang Y, Ma H, Liu T, Fuchs J, Li PK, Lu J, Li C, Lin J. STAT3 as a potential therapeutic target in ALDH+ and CD44+/CD24+ stem cell-like pancreatic cancer cells. Int J Oncol. 2016;49(6):2265-74

26. Lin L, Fuchs J, Li C, Olson V, Bekaii-Saab T, Lin J. STAT3 signaling pathway is necessary for cell survival and tumorsphere forming capacity in ALDH(+ )/CD133(+) stem cell-like human colon cancer cells. Biochem Biophys Res Commun. 2011:416(3-4):246-51.

27. Morgan RG, Mortensson E, Legge DN, Gupta B, Collard TJ, Greenhough A, Williams AC. LGR5 expression is regulated by EGF in early colorectal adenomas and governs EGFR inhibitor sensitivity. Br J Cancer. 2017;

28. Ahmad R, Kumar B, Chen Z, Chen X, Muller D, Lele SM, Washington MK, Batra SK, Dhawan P, Singh AB. Loss of claudin-3 expression induces IL6/ gp130/Stat3 signaling to promote colon cancer malignancy by hyperactivating Wnt/beta-catenin signaling. Oncogene. 2017;36(47):6592-604.

29. Ibrahem S, Al-Ghamdi S, Baloch K, Muhammad B, Fadhil W, Jackson D, Nateri AS, Ilyas M. STAT3 paradoxically stimulates beta-catenin expression but inhibits beta-catenin function. Int J Exp Pathol. 2014;95(6):392-400.

30. Chen MW, Yang ST, Chien MH, Hua KT, Wu CJ, Hsiao SM, Lin H, Hsiao M, Su $J$, Wei LH. The STAT3-miRNA-92-Wnt signaling pathway regulates spheroid formation and malignant progression in ovarian Cancer. Cancer Res. 2017;77(8):1955-67

31. Hao J, Li TG, Qi X, Zhao DF, Zhao GQ. WNT/beta-catenin pathway upregulates Stat3 and converges on LIF to prevent differentiation of mouse embryonic stem cells. Dev Biol. 2006;290(1):81-91.

32. Phesse TJ, Buchert $M$, Stuart E, Flanagan DJ, Faux M, Afshar-Sterle S, Walker F, Zhang HH, Nowell CJ, Jorissen R, Tan CW, Hirokawa Y, Eissmann MF, Poh AR, Malaterre J, Pearson HB, Kirsch DG, Provero P, Poli V, Ramsay RG, Sieber O, Burgess AW, Huszar D, Vincan E, Ernst M. Partial inhibition of gp130-JakStat3 signaling prevents Wnt-beta-catenin-mediated intestinal tumor growth and regeneration. Sci Signal. 2014;7(345):ra92.

33. Yamashina K, Yamamoto H, Chayama K, Nakajima K, Kikuchi A. Suppression of STAT3 activity by Duplin, which is a negative regulator of the Wnt signal. J Biochem. 2006;139(2):305-14.

34. Monteiro J, Fodde R. Cancer stemness and metastasis: therapeutic consequences and perspectives. Eur J Cancer. 2010;46(7):1198-203.

35. Dittmar T, Heyder C, Gloria-Maercker E, Hatzmann W, Zanker KS. Adhesion molecules and chemokines: the navigation system for circulating tumor (stem) cells to metastasize in an organ-specific manner. Clin Exp Metastasis. 2008;25(1):11-32.

36. Zhuang J, Shen L, Yang L, Huang X, Lu Q, Cui Y, Zheng X, Zhao X, Zhang D, Huang $\mathrm{R}$, Guo H, Yan J. TGFbeta1 promotes gemcitabine resistance through regulating the LncRNA-LET/NF90/miR-145 signaling Axis in bladder Cancer. Theranostics. 2017;7(12):3053-67.

37. Cheng CC, Chang J, Huang SC, Lin HC, Ho AS, Lim KH, Chang CC, Huang L, Chang YC, Chang YF, Wu CW. YM155 as an inhibitor of cancer stemness simultaneously inhibits autophosphorylation of epidermal growth factor receptor and G9a-mediated stemness in lung cancer cells. PLoS One. 2017;12(8):e0182149

38. Vlashi E, Kim K, Lagadec C, Donna LD, McDonald JT, Eghbali M, Sayre JW, Stefani E, McBride W, Pajonk F. In vivo imaging, tracking, and targeting of cancer stem cells. J Natl Cancer Inst. 2009;101(5):350-9.

39. Shih BB, Chang YF, Cheng CC, Yang HJ, Chang KW, Ho AS, Lin HC, Yeh C, Chang CC. SPECT imaging evaluation of (111)indium-chelated cetuximab for diagnosing EGFR-positive tumor in an HCT-15-induced colorectal xenograft. J Chin Med Assoc. 2017;80(12):766-73.

40. Messersmith WA, Ahnen DJ. Targeting EGFR in colorectal cancer. N Engl J Med. 2008:359(17):1834-6.

41. Shih YH, Peng CL, Lee SY, Chiang PF, Yao CJ, Lin WJ, Luo TY, Shieh MJ. 111 In-cetuximab as a diagnostic agent by accessible epidermal growth factor (EGF) receptor targeting in human metastatic colorectal carcinoma. Oncotarget. 2015;6(18):16601-10.
42. Feng Y, Dai X, Li X, Wang H, Liu J, Zhang J, Du Y, Xia L. EGF signalling pathway regulates colon cancer stem cell proliferation and apoptosis. Cell Prolif. 2012;45(5):413-9.

43. Shostak K, Chariot A. EGFR and NF-kappaB: partners in cancer. Trends Mol Med. 2015;21(6):385-93.

44. De Roock W, Claes B, Bernasconi D, De Schutter J, Biesmans B, Fountzilas G, Kalogeras KT, Kotoula V, Papamichael D, Laurent-Puig P, Penault-Llorca F, Rougier P, Vincenzi B, Santini D, Tonini G, Cappuzzo F, Frattini M, Molinari F, Saletti P, De Dosso S, Martini M, Bardelli A, Siena S, Sartore-Bianchi A, Tabernero J, Macarulla T, Di Fiore F, Gangloff AO, Ciardiello F, Pfeiffer P, Qvortrup C, Hansen TP, Van Cutsem E, Piessevaux H, Lambrechts D, Delorenzi M, Tejpar S. Effects of KRAS, BRAF, NRAS, and PIK3CA mutations on the efficacy of cetuximab plus chemotherapy in chemotherapyrefractory metastatic colorectal cancer: a retrospective consortium analysis. Lancet Oncol. 2010;11(8):753-62.

45. Hsu HC, Thiam TK, Lu YJ, Yeh CY, Tsai WS, You JF, Hung HY, Tsai CN, Hsu A, Chen HC, Chen SJ, Yang TS. Mutations of KRAS/NRAS/BRAF predict cetuximab resistance in metastatic colorectal cancer patients. Oncotarget. 2016;7(16):22257-70

46. Novotny L, Al-Tannak NF, Hunakova L. Protein synthesis inhibitors of natural origin for CML therapy: semisynthetic homoharringtonine (Omacetaxine mepesuccinate). Neoplasma. 2016:63(4):495-503.

47. Cao W, Liu Y, Zhang R, Zhang B, Wang T, Zhu X, Mei L, Chen H, Zhang H, Ming $P$, Huang L. Homoharringtonine induces apoptosis and inhibits STAT3 via IL-6/JAK1/STAT3 signal pathway in Gefitinib-resistant lung cancer cells. Sci Rep. 2015:5:8477.

48. Cao Y. Multifarious functions of PDGFs and PDGFRs in tumor growth and metastasis. Trends Mol Med. 2013:19(8):460-73.

49. Heldin $\mathrm{CH}$. Autocrine PDGF stimulation in malignancies. Ups J Med Sci. 2012;117(2):83-91.

50. Pattabiraman DR, Weinberg RA. Tackling the cancer stem cells - what challenges do they pose? Nat Rev Drug Discov. 2014;13(7):497-512.

\section{Ready to submit your research? Choose BMC and benefit from:}

- fast, convenient online submission

- thorough peer review by experienced researchers in your field

- rapid publication on acceptance

- support for research data, including large and complex data types

- gold Open Access which fosters wider collaboration and increased citations

- maximum visibility for your research: over $100 \mathrm{M}$ website views per year

At BMC, research is always in progress.

Learn more biomedcentral.com/submissions 